\title{
Enantioselective Synthesis of Medium-Sized Ring-Bridged Oxabicycles by Ring-Closing Metathesis
}

\author{
Pedro de Armas, ${ }^{*[a]}$ Fernando García-Tellado, ${ }^{*|a|}$ and José Juan Marrero-Tellado*|b|
}

Dedicated to Professor Marcial Moreno-Mañas on the occasion of his 60th birthday

Keywords: Metathesis / Glycosides / Cyclizations / Carbohydrates

A flexible strategy is described for the enantioselective construction of both optical antipodes of the oxabicyclo[4.2.1]-, -[5.2.1]-, and -[6.2.1]alkenes by a ring-closing metathesis reaction of the suitable 2,5-cis-dialkenyltetrahydrofuran derivatives.

\section{Introduction}

Ring-closing metathesis (RCM) catalysed by carbene transition metal complexes ${ }^{[1,2]}$ has become one of the most popular synthetic methods for the formation of a $\mathrm{C}-\mathrm{C}$ bond. Commercially available Grubbs' catalyst ${ }^{[3]}$ is widely used because of its tolerance of a wide range of functional groups and its easy bench handling. While the RCM reaction has found a wide use in the construction of medium ring-sized carbo- and heterocycles, its use in the construction of medium-sized bridged compounds is scarce. ${ }^{[4]}$ In this paper we report on a flexible strategy for the enantioselective synthesis of the medium-sized bridged oxabicyclo $[n$.2.1]alkenes $\mathbf{V}$ and $\boldsymbol{e n t} \mathbf{- V}(n=4,5,6)$ (Scheme 1) by an RCM reaction of the conformationally constrained dienes IV and ent-IV, respectively. Bridged oxabicyclic compounds are not only a synthetic target in their own right, but also because of their use as templates and building blocks in organic synthesis. ${ }^{[5]}$ In addition, the bridged 11-oxabicyclo[6.2.1]undecane is a central and common sub-structural motif of the cembranoids, ${ }^{[6]}$ a pharmacologically important family of marine metabolites with a wide spectrum of biological activity.

\section{Results and Discussion}

The strategy is outlined in Scheme 1. We proposed to build the enantiomeric medium-sized bridged oxabicycles $\mathbf{V}$ and $\boldsymbol{e n t} \mathbf{- V}$ through the ring-closing metathesis reactions of dienes IV and ent-IV, respectively. The 2,5-cis geometry of

[a] Instituto de Productos Naturales y Agrobiología, CSIC,

Avda. Astrofísico Francisco Sánchez 3, 38206 La Laguna,

Tenerife, Spain

Fax: (internat.) + 34-922/260135

E-mail: fgarcia@ipna.csic.es; parmas@ipna.csic.es

[b] Instituto Universitario de Bio-Orgánica, "Antonio González", Universidad de La Laguna,

Avda. Astrofísico Francisco Sánchez 2, 38206 La Laguna, Tenerife, Spain

Fax: (internat.) + 34-922/260135

E-mail: jtellado@ull.es
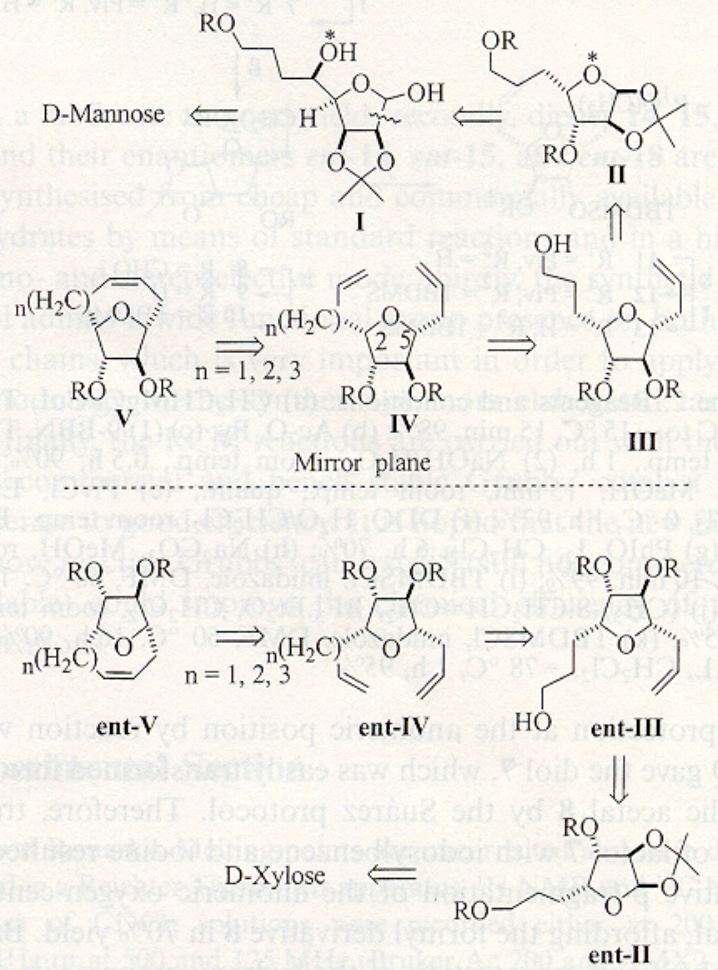

Scheme 1. Retrosynthetic analysis for the synthesis of both antipodes of medium-sized bridged oxabicyclo[n.2.1] alkenes $\mathbf{V}$ and ent$\mathbf{V}(n=1,2,3)$

the alkenyl chains must bring the diene termini into close proximity and so reduce the entropic cost associated with the ring-closing process. The exo-directed allylation ${ }^{[7]}$ of the bicyclic acetals II and ent-II should permit access to olefins III and ent-III, respectively, as diastereomerically pure compounds. The bicyclic acetal II is obtained from diol I through the Suarez protocol,,$^{[8,9]}$ which relocates the original anomeric position of D-mannose to the new one created by $\beta$-fragmentation at the anomeric position, and traps the intermediate by the secondary hydroxy group placed on the chain (*-marked oxygen atom). Finally, the enantiom- 
eric acetal ent-II is directly obtained from D-xylose by simple protecting groups manipulation.

\section{Synthesis of the Dienes}

Epoxide $\mathbf{1}^{[10]}$ (Scheme 2) was treated with vinylmagnesium chloride in the presence of $\mathrm{CuI}^{[11]}$ to give alcohol 2 in $98 \%$ yield. Protection and oxidative hydroboration gave the alcohol 4 in $90 \%$ yield. Hydrolysis and regioselective protection of the primary hydroxy group as its pivaloyl ester gave the alcohol 6 in $92 \%$ yield.

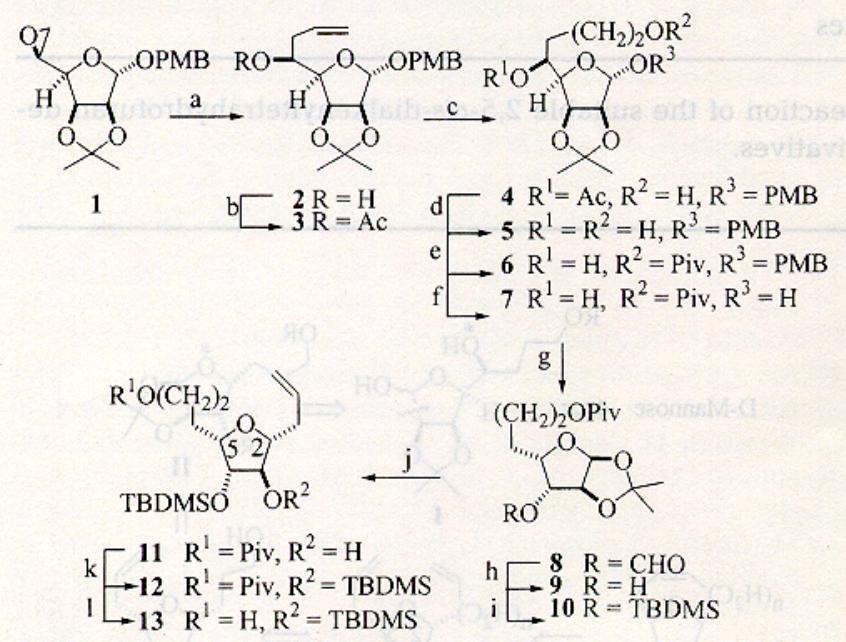

Scheme 2. Reagents and conditions: (a) $\mathrm{CH}_{2} \mathrm{CHMgCl} / \mathrm{CuI}$, THF $-30{ }^{\circ} \mathrm{C}$ to $-15^{\circ} \mathrm{C}, 15 \mathrm{~min}, 98 \%$; (b) $\mathrm{Ac}_{2} \mathrm{O}, \mathrm{Py}$; (c) (1) 9-BBN, THF, room temp., $1 \mathrm{~h}$, (2) $\mathrm{NaOH} / \mathrm{H}_{2} \mathrm{O}_{2}$, room temp., $0.5 \mathrm{~h}, 90 \%$; (d) $\mathrm{KOH}, \mathrm{MeOH}, 15 \mathrm{~min}$, room temp., quant.; (e) $\mathrm{PivCl}, \mathrm{Et}_{3} \mathrm{~N}$, $\mathrm{CH}_{2} \mathrm{Cl}_{2}, 0^{\circ} \mathrm{C}, 1 \mathrm{~h}, 92 \%$; (f) DDQ, $\mathrm{H}_{2} \mathrm{O} / \mathrm{CH}_{2} \mathrm{Cl}_{2}$, room temp., $16 \mathrm{~h}$, $91 \%$; (g) PhIO, $\mathrm{I}_{2}, \mathrm{CH}_{2} \mathrm{Cl}_{2}, 6 \mathrm{~h}, 70 \%$; (h) $\mathrm{Na}_{2} \mathrm{CO}_{3}, \mathrm{MeOH}$, room temp., $10 \mathrm{~min}, 99 \%$; (i) TBDMSCl, imidazole, DMF, $60{ }^{\circ} \mathrm{C}, 16 \mathrm{~h}$, $91 \%$. (j) $\left(\mathrm{CH}_{3}\right)_{3} \mathrm{SiCH}_{2} \mathrm{CH}=\mathrm{CH}_{2}, \mathrm{BF}_{3} \cdot \mathrm{Et}_{2} \mathrm{O}, \mathrm{CH}_{2} \mathrm{Cl}_{2}$, room temp. $6 \mathrm{~h}, 85 \%$; (k) TBDMSCl, imidazole, DMF, $60{ }^{\circ} \mathrm{C}, 16 \mathrm{~h}, 90 \%$; (1) DIBAL, $\mathrm{CH}_{2} \mathrm{Cl}_{2},-78{ }^{\circ} \mathrm{C}, 1 \mathrm{~h}, 95 \%$

Deprotection at the anomeric position by reaction with DDQ gave the diol 7, which was easily transformed into the bicyclic acetal 8 by the Suárez protocol. Therefore, treatment of lactol 7 with iodosylbenzene and iodine resulted in oxidative $\beta$-fragmentation of the anomeric oxygen-centred radical, affording the formyl derivative 8 in $70 \%$ yield. Basic hydrolysis and reprotection of the hydroxy group as its tertbutyldimethylsilyl ether gave the acetal 10 in a yield of $91 \%$. The exo-directed allylation ${ }^{[7]}$ of this bicyclic acetal gave the alkene 11 as a unique diastereomer in $85 \%$ yield. Protection of the secondary hydroxy group as its tert-butyldimethylsilyl ether followed by DIBAL deprotection of the primary pivaloyl ester gave the alcohol 13 in a yield of $86 \%$. Synthetic manipulation of the primary hydroxy group of $\mathbf{1 3}$ allowed the installation of the required second alkenyl chain (Scheme 3). Therefore, Grieco elimination of the primary hydroxy group gave diene $\mathbf{1 4}$ in a $75 \%$ yield. Swern oxidation, followed by a Wittig olefination efficiently transformed 13 into the diene $15(70 \%)$. Finally, mesylate formation, cyanide displacement, DIBAL reduction of the cyanide group to the imine, followed by hydrolysis to the aldehyde, and Wittig olefination afforded diene 18 in a $40 \%$ overall yield.

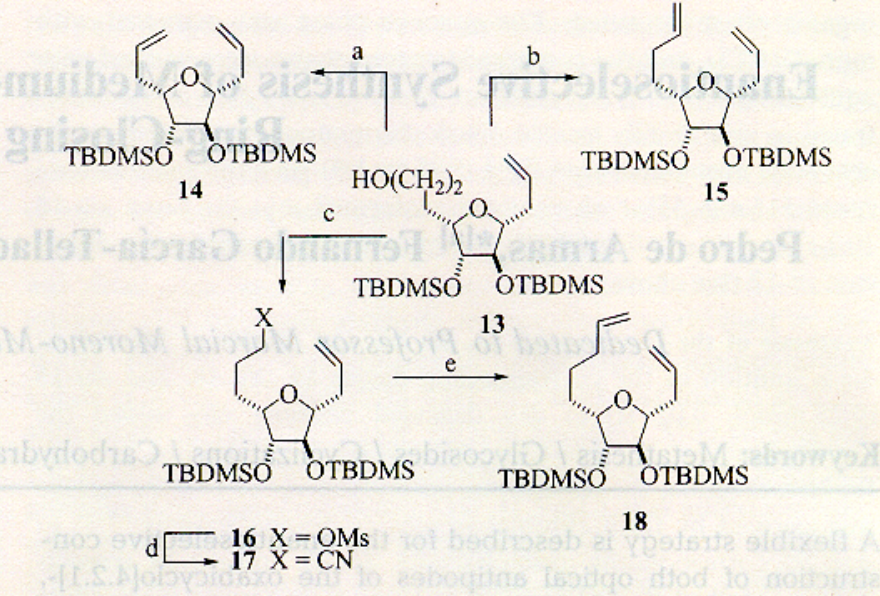

Scheme 3. Reagents and conditions: (a) (1) $o-\mathrm{NO}_{2} \mathrm{C}_{6} \mathrm{H}_{4} \mathrm{SeCN}$, $\mathrm{Bu}_{3} \mathrm{P}$, THF, room temp., $16 \mathrm{~h}$, (2) $\mathrm{H}_{2} \mathrm{O}_{2}$, THF, $0{ }^{\circ} \mathrm{C}, 3 \mathrm{~h}, 75 \%$ two steps; (b) (1) Swern oxidation, (2) $\mathrm{Ph}_{3} \mathrm{PCH}_{3} \mathrm{I}, n \mathrm{BuLi}$, THF, $0{ }^{\circ} \mathrm{C}$, $1 \mathrm{~h}, 70 \%$ two steps; (c) $\mathrm{MsCl}, \mathrm{Et}_{3} \mathrm{~N}, \mathrm{CH}_{2} \mathrm{Cl}_{2}, 0{ }^{\circ} \mathrm{C}, 30 \mathrm{~min}, 100 \%$; (d) $\mathrm{KCN}, \mathrm{DMF}, 50^{\circ} \mathrm{C}, 10 \mathrm{~h}, 68 \%$; (e) (1) DIBAL, $\mathrm{CH}_{2} \mathrm{Cl}_{2},-78$ ${ }^{\circ} \mathrm{C}, 1 \mathrm{~h},(2)$ aq. $\mathrm{HCl}(1 \mathrm{~N}),(3) \mathrm{Ph}_{3} \mathrm{PCH}_{3} \mathrm{I}, n \mathrm{BuLi}$, THF, $0{ }^{\circ} \mathrm{C}, 1 \mathrm{~h}$, $56 \%$ for the three steps

As the representative example of the enantiomeric series, we chose the readily accessible diene $\boldsymbol{e n t}-\mathbf{1 5}$, which was easily synthesised from commercial 1,2-O-isopropylidene-D-xylofuranose (19) through the synthetic sequence outlined in the Scheme 4. Selective monoprotection of the secondary hydroxy group of $19^{[12]}$ as its tert-butyldimethylsilyl ether gave the alcohol 20 in a $54 \%$ yield. Treatment of 20 with molecular iodine and triphenylphosphane/imidazole gave the iodine derivative 21, which was transformed into alkene 22 by radical allylation with allyltri- $n$-butylstannane and azobis(isobutyronitrile) $(86 \%)$. The exo-directed allylation of this bicyclic acetal followed by hydroxy group protection gave the diene ent-15 as the unique diastereomer in $55 \%$ of yield.

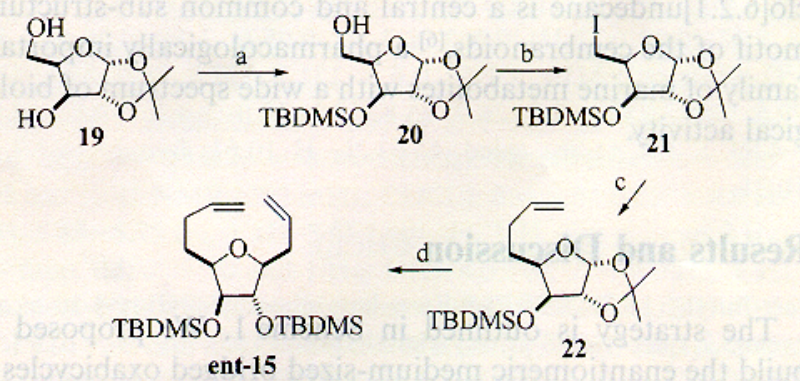

Scheme 4. Reagents and conditions: (a) (1) $\left(\mathrm{Bu}_{3} \mathrm{Sn}\right)_{2} \mathrm{O}$, toluene, reflux, $12 \mathrm{~h}$ and then $p \mathrm{MeOBzCl}$, reflux, $62 \mathrm{~h}, 80 \%$; (2) TBDMSCl, imidazole, DMF, $60^{\circ} \mathrm{C}, 16 \mathrm{~h}, 85 \%$; (3) DDQ, $\mathrm{CH}_{2} \mathrm{Cl}_{2} / \mathrm{H}_{2} \mathrm{O}$, room temp., $2 \mathrm{~h}, 80 \%$; (b) $\mathrm{I}_{2}, \mathrm{Ph}_{3} \mathrm{P}$, imidazole, $\mathrm{C}_{6} \mathrm{H}_{6}, 2 \mathrm{~h}$, reflux, $90 \%$; (c) $n \mathrm{Bu}_{3} \mathrm{SnCH}_{2} \mathrm{CH}=\mathrm{CH}_{2}$, AIBN cat, toluene, reflux, $16 \mathrm{~h}, 86 \%$; (d) (1) $\left(\mathrm{CH}_{3}\right)_{3} \mathrm{SiCH}_{2} \mathrm{CH}=\mathrm{CH}_{2}, \mathrm{BF}_{3} \cdot \mathrm{Et}_{2} \mathrm{O}, \mathrm{CH}_{2} \mathrm{Cl}_{2}$, room temp., $3 \mathrm{~h}$; (2) TBDMSCl, imidazole, DMF, $60^{\circ} \mathrm{C}, 16 \mathrm{~h}, 55 \%$ two steps

\section{Ring-Closing Metathesis Reactions}

Initial metathesis reactions were carried out with the diene 14 to obtain the 9-oxabicyclo[4.2.1]non-3-ene (23) 
Table 1. Conditions for ring-closing metathesis of dienes 14,15 , ent-15, and 18

\begin{tabular}{|c|c|c|c|c|c|c|c|c|}
\hline Entry & Substrate & Solvent & $T\left[{ }^{\circ} \mathrm{C}\right]$ & $t[\mathrm{~h}]$ & {$[\mathrm{M}]^{[\mathrm{a}][\mathrm{b}]}$} & Yield (\%) & $\operatorname{RSM}^{[c]}(\%)$ & Product \\
\hline 1 & 14 & $\mathrm{CH}_{2} \mathrm{Cl}_{2}$ & room temp. & 24 & A & - & 100 & \\
\hline 2 & 14 & $\mathrm{CH}_{2} \mathrm{Cl}_{2}$ & reflux & 24 & A & - & 100 & - \\
\hline 3 & 14 & $\mathrm{C}_{6} \mathrm{H}_{6}$ & 40 & 16 & A & - & 100 & - \\
\hline 4 & 14 & $\mathrm{C}_{6} \mathrm{H}_{6}$ & reflux & 16 & A & 64 & 8 & 23 \\
\hline 5 & 14 & $\mathrm{C}_{6} \mathrm{H}_{6}$ & reflux & 16 & C & 61 & 7 & 23 \\
\hline 6 & 15 & $\mathrm{CH}_{2} \mathrm{Cl}_{2}$ & room temp. & 24 & A & - & 100 & - \\
\hline 7 & 15 & $\mathrm{CH}_{2} \mathrm{Cl}_{2}$ & reflux & 24 & A & - & 100 & - \\
\hline 8 & 15 & $\mathrm{C}_{2} \mathrm{H}_{4} \mathrm{Cl}_{2}$ & reflux & 16 & A & 24 & 7 & 24 \\
\hline 9 & 15 & $\mathrm{C}_{6} \mathrm{H}_{6}$ & reflux & 16 & A & 45 & 18 & 24 \\
\hline 10 & 15 & $\mathrm{C}_{6} \mathrm{H}_{6}$ & reflux & 16 & B & 41 & 12 & 24 \\
\hline 11 & 15 & $\mathrm{C}_{6} \mathrm{H}_{6}$ & reflux & 16 & $\mathrm{C}$ & 46 & 3 & 24 \\
\hline 12 & ent-15 & $\mathrm{C}_{6} \mathrm{H}_{6}$ & reflux & 16 & A & 40 & 10 & ent-24 \\
\hline 13 & 18 & $\mathrm{C}_{6} \mathrm{H}_{6}$ & reflux & 16 & A & 53 & 8 & 25 \\
\hline 14 & 18 & $\mathrm{C}_{6} \mathrm{H}_{6}$ & reflux & 16 & B & 40 & 9 & 25 \\
\hline 15 & 18 & $\mathrm{C}_{6} \mathrm{H}_{6}$ & reflux & 16 & C & 50 & 15 & 25 \\
\hline
\end{tabular}

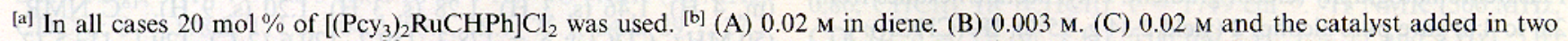
portions at eight hour intervals. ${ }^{[c]}$ RSM $=$ Recovered Starting Material.

(Scheme 5). After attempting to modify several experimental conditions including solvent, catalyst charge, ${ }^{[13]}$ and temperature (Table 1, Entries 1-5), refluxing benzene ( 0.02 M) with a $20 \mathrm{~mol} \%$ of Grubbs' catalyst charge were found to be the best conditions, affording $\mathbf{2 4}$ in $64 \%$ yield. Remarkably, in spite of the known difficulty in forming eightmembered rings by this reaction, ${ }^{[4 a, 4 c]}$ dienes 15 and ent -15 gave the 10-oxabicyclo[5.2.1]dec-3-enes $\mathbf{2 4}$ and ent-24 in a $45 \%$ and $40 \%$ yield, respectively (Entries 9 and 12 ). Unfortunately, the efficiency of the reaction could not be improved by changes in the experimental conditions (Entries $6-11)$.

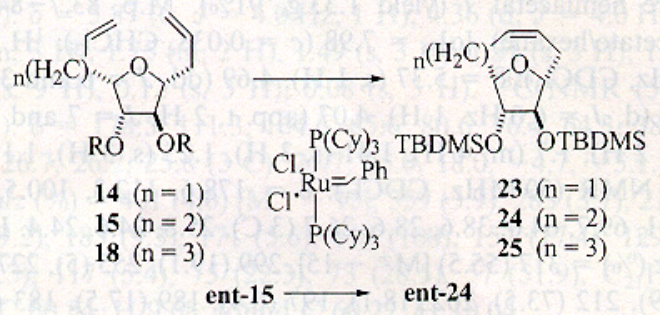

Scheme 5. Ring-closing metathesis reaction of dienes 14,15 , ent15 , and 18 to give the oxabicycles 23,24 , ent-24, and 25 .

Diene 18 also underwent the RCM reaction with acceptable efficiency, affording the $(1 S, 8 R, 9 S, 10 S)$-9, 10-di-tert-butoxy-11-oxabicyclo[6.2.1]undec-3-ene (25) in $53 \%$ yield (Entries 13-15). Although this 53\% yield is susceptible to improvement by the use of other catalysts, ${ }^{[14]}$ this is still good enough for synthetic use.

\section{Conclusion}

Some features of this methodology are noteworthy: Firstly, it opens a synthetic access to the medium-sized oxabicyclo[4.2.1]non-3-ene, -[5.2.1]dec-3-ene, and -[6.2.1]undec-3-ene in both their enantiomerically pure forms and with a moderate to good yield; secondly, dienes 14,15 , and 18 and their enantiomers ent-14, ent -15 , and ent-18 are easily synthesised from cheap and commercially available carbohydrates by means of standard reactions and in a highly chemo- and stereoselective mode; thirdly, the synthetic protocol admits a wide functional group presence on both alkenyl chains, which is very important in order to apply this methodology to the synthesis of more elaborated targets; and finally, the RCM reactions are carried out with the aid of a commercial and bench stable Grubbs' catalyst with moderate to good efficiency. It is hoped that the new family of more reactive Grubbs' catalysts ${ }^{[14]}$ (still not commercially available) could improve the chemical efficiency of these cyclizations.

\section{Experimental Section}

General Remarks: Melting points are uncorrected and were determined in a Reichter Thermovar apparatus. ${ }^{1} \mathrm{H}$ NMR and ${ }^{13} \mathrm{C}$ NMR spectra of $\mathrm{CDCl}_{3}$ solutions were recorded either at 200 and $50 \mathrm{MHz}$ or at 500 and $125 \mathrm{MHz}$ (Bruker Ac 200 and AMX2-500), respectively. FT-IR spectra were measured in chloroform solutions using a Shimadzu IR-408 spectrophotometer. Mass spectra (low resolution) (EI/CI) were obtained with a Hewlett-Packard 5995 gas chromatograph/mass spectrometer. High-resolution mass spectra were recorded with a Micromass Autospec mass spectrometer. Microanalyses were performed with a Fisons Instruments EA 1108 carbon, hydrogen, and nitrogen analyser. Optical rotations were determined at room temperature with a Perkin -Elmer 241 polarimeter and are referenced to the D-line of sodium. Analytical thin-layer chromatography plates used were E. Merck Brinkman UV-active silica gel (Kieselgel 60 F254) on aluminium. Flash column chromatography was carried out with E. Merck silica gel 60 (particle size less than $0.020 \mathrm{~mm}$ ) using appropriate mixtures of ethyl acetate and hexanes as eluent. All reactions were performed in oven-dried glassware under nitrogen unless otherwise stated in the text. Tetrahydrofuran, benzene, and toluene were distilled from sodium metal/ benzophenone ketyl. Dichloromethane, dimethyl sulfoxide, dimethylformamide, and triethylamine were distilled from $\mathrm{CaH}_{2}$. 
Bis(tricyclohexylphosphane)benzylideneruthenium(IV) dichloride (Grubbs' catalyst) was purchased from Strem Chemicals, and used under nitrogen with standard Schlenk techniques.

4-Methoxybenzyl 6,7,8-Trideoxy-2,3-O-(1-methylethylidene)- $\alpha$-Dmanno-oct-7-enofuranoside (2): In an oven-dried round-bottomed flask was placed $\mathrm{CuI}(1.29 \mathrm{~g}, 6.76 \mathrm{mmol})$ and the flask was purged with nitrogen. Dry THF $(20 \mathrm{~mL})$ was added and the suspension was cooled at $-30{ }^{\circ} \mathrm{C}$. Vinylmagnesium chloride (1.7 $\mathrm{M}$ in THF) $(8 \mathrm{~mL}, 13.6 \mathrm{mmol})$ was added and the mixture was stirred at -30 ${ }^{\circ} \mathrm{C}$ for $15 \mathrm{~min}$. Then, epoxide $1(2.18 \mathrm{~g}, 6.8 \mathrm{mmol})$ in dry THF $(40 \mathrm{~mL})$ was added via cannula and the mixture was slowly warmed from $-30^{\circ} \mathrm{C}$ to $-10^{\circ} \mathrm{C}$ during $2 \mathrm{~h}$. Aqueous saturated $\mathrm{NH}_{4} \mathrm{Cl}$ was added to destroy the reagent excess and the mixture was transferred to a decantation funnel with the aid of more ethyl acetate. The organic phase was washed with $\mathrm{H}_{2} \mathrm{O}(3 \times), 1 \mathrm{~N} \mathrm{HCl}(3 \times)$, aqueous saturated $\mathrm{NaHCO}_{3}(3 \times)$, and aqueous saturated $\mathrm{NaCl}(3 \times)$, dried with $\mathrm{Na}_{2} \mathrm{SO}_{4}$, filtered and concentrated to give a gummy residue. Flash chromatography (eluent gradient: ethyl acetate/hexanes from $2: 8$ to $4: 6$ ) gave pure alcohol 2 (yield $2.34 \mathrm{~g}, 95 \%$ ). IR $\left(\mathrm{CHCl}_{3}\right): \tilde{v}=$ $3514,3007 \mathrm{~cm}^{-1} .{ }^{1} \mathrm{H}$ NMR $\left(200 \mathrm{MHz}, \mathrm{CDCl}_{3}\right): \delta=7.25(\mathrm{~d}, J=$ $9.0 \mathrm{~Hz}, 2 \mathrm{H}), 6.87$ (d, $J=9.0 \mathrm{~Hz}, 2 \mathrm{H}), 5.87(\mathrm{~m}, 1 \mathrm{H}), 5.19$ (dd, $J=7$ and $2 \mathrm{~Hz}, 1 \mathrm{H}), 5.14(\mathrm{~s}, 1 \mathrm{H}, \mathrm{H}-1), 5.13$ (dd, $J=10$ and $2 \mathrm{~Hz}, 1 \mathrm{H}), 4.75(\mathrm{dd}, J=6$ and $3 \mathrm{~Hz}, 1 \mathrm{H}), 4.64(\mathrm{~d}, J=6.0 \mathrm{~Hz}, 1$ $\mathrm{H}), 4.61$ and $4.41(\mathrm{~d}, 1 \mathrm{H}$ each, $J=11.0 \mathrm{~Hz}), 4.11(\mathrm{dd}, J=11$ and $4 \mathrm{~Hz}, 1 \mathrm{H}$ ), 3.89 (app. t, $1 \mathrm{H}, J=6$ and $4 \mathrm{~Hz}$ ), $3.80(\mathrm{~s}, 3 \mathrm{H}), 2.41$ $(\mathrm{m}, 1 \mathrm{H}), 1.47(\mathrm{~s}, 3 \mathrm{H}), 1.29(\mathrm{~s}, 3 \mathrm{H}) \cdot{ }^{13} \mathrm{C} \mathrm{NMR}\left(50 \mathrm{MHz}, \mathrm{CDCl}_{3}\right)$ : $\delta=159.2,134.3,129.6$ (2 C), 129.1, 117.3, 113.6 (2 C), 112.4, $104.5,85.3,80.8,80.2,69.3,66.4,55.0,37.7,25.7,24.2$. LRMS (EI): $m / z(\%)=350(0.9)\left[\mathrm{M}^{+}\right], 335(1.4), 229(2.9), 171(8.4), 121$ (100), 91 (2), 83 (3.6), 69 (2.7). HRMS (EI): calcd. for $\mathrm{C}_{19} \mathrm{H}_{26} \mathrm{O}_{6}$ 350.172939 , found 350.174675 . The alcohol was acetylated with $\mathrm{Ac}_{2} \mathrm{O} / \mathrm{Py}$ to give the acetate 3 , which was directly used in the next experiment.

\begin{abstract}
4-Methoxybenzyl 5-O-Acetyl-6,7-dideoxy-2,3-O-(1-methylethylidene)- $\boldsymbol{\alpha}$-D-manno-octofuranoside (4): Alkene $3(1.88 \mathrm{~g}, 4.8 \mathrm{mmol})$ in dry THF $(20 \mathrm{~mL})$ was stirred with $9-\mathrm{BBN}(0.5 \mathrm{M}$ THF $)(19 \mathrm{~mL}$, $9.5 \mathrm{mmol}$ ) at room temperature for $45 \mathrm{~min} .3 \mathrm{~N} \mathrm{NaOH}$ solution $(1.6 \mathrm{~mL})$ and $30 \% \mathrm{H}_{2} \mathrm{O}_{2}(3 \mathrm{~mL}, 27 \mathrm{mmol})$ were cautiously added and the resulting mixture was stirred for $30 \mathrm{~min}$. The excess reagent was destroyed by addition of aqueous saturated $\mathrm{NaHSO}_{3}$ solution, and the solvent was distilled off. Ethyl acetate was added and the solution was transferred to a decantation funnel. The organic phase was washed with aqueous saturated solutions of $\mathrm{NaHSO}_{3}$ and $\mathrm{NaCl}$, dried with $\mathrm{Na}_{2} \mathrm{SO}_{4}$, filtered and concentrated to give a gummy residue. Flash chromatography (eluent gradient: ethyl acetate/hexanes from $2: 8$ to $6: 4$ ) gave pure 4 (yield $1.78 \mathrm{~g}, 90 \%$ ). $[\alpha]_{\mathrm{D}}=$ $43\left(c=0.221, \mathrm{CHCl}_{3}\right)$. IR $\left(\mathrm{CHCl}_{3}\right): \tilde{v}=3500,1735 \mathrm{~cm}^{-1} \cdot{ }^{1} \mathrm{H}$ NMR $\left(200 \mathrm{MHz}, \mathrm{CDCl}_{3}\right): \delta=7.24(\mathrm{~d}, J=9.0 \mathrm{~Hz}, 2 \mathrm{H}), 6.88(\mathrm{~d}, J=$ $9.0 \mathrm{~Hz}, 2 \mathrm{H}), 5.36(\mathrm{~m}, 1 \mathrm{H}), 5.08(\mathrm{~s}, 1 \mathrm{H}), 4.71(\mathrm{dd}, J=6$ and $4 \mathrm{~Hz}$, $1 \mathrm{H}), 4.62(\mathrm{~d}, J=6.0 \mathrm{~Hz}, 1 \mathrm{H}), 4.58$ and $4.40(\mathrm{~d}$ each, $J=11.0 \mathrm{~Hz}$, $2 \mathrm{H}$ each), 4.00 (dd, $J=9$ and $4 \mathrm{~Hz}, 1 \mathrm{H}), 3.81(\mathrm{~s}, 3 \mathrm{H}), 3.7(\mathrm{~m}, 1$ $\mathrm{H}), 2.12$ (s, $3 \mathrm{H}), 1.44(\mathrm{~s}, 3 \mathrm{H}), 1.29(\mathrm{~s}, 3 \mathrm{H}),{ }^{13} \mathrm{C}$ NMR $(50 \mathrm{MHz}$, $\left.\mathrm{CDCl}_{3}\right): \delta=170.5,159.1,129.6(2 \mathrm{C}), 129.2,113.5$ (2 C), 104.8, $85.1,80.9,79.5,71.5,68.4,61.7,55.1,27.7,26.8,25.9,24.8,21.0$. LRMS (EI): $m / z(\%)=410(1.6)\left[\mathrm{M}^{+}\right], 257(2.3), 242(0.8), 231$ (9.4), $213(0.9), 197$ (1.8), $185(4), 171$ (6.7), $155(2.6), 143(8), 135$ (8.2), 125 (15.8), 121 (100). $\mathrm{C}_{21} \mathrm{H}_{30} \mathrm{O}_{8}$ : calcd. C 61.45, $\mathrm{H}$ 7.37; found C $61.586, \mathrm{H} 7.556$.
\end{abstract}

4-Methoxybenzyl 6,7-Dideoxy-8-O-(2,2-dimethylpropanoyl)-2,3-O(1-methylethylidene)- $\alpha$-D-manno-octofuranoside (6): Acetate 4 $(1.78 \mathrm{~g}, 4.4 \mathrm{mmol})$ was stirred with $\mathrm{KOH}(3 \mathrm{~g}, 54 \mathrm{mmol})$ in $\mathrm{MeOH}$ $(45 \mathrm{~mL})$ for $15 \mathrm{~min}$. The solvent was distilled off and the residue was taken up in $\mathrm{CH}_{2} \mathrm{Cl}_{2} / \mathrm{H}_{2} \mathrm{O}$. The mixture was decanted and the aqueous phase extracted with $\mathrm{CH}_{2} \mathrm{Cl}_{2}$. The combined organic phases were washed with aqueous saturated $\mathrm{NaCl}$, dried with $\mathrm{Na}_{2} \mathrm{SO}_{4}$, filtered, and concentrated to give diol $\mathbf{5}$ as a crystalline solid. The crude diol 5 in dry $\mathrm{CH}_{2} \mathrm{Cl}_{2}(30 \mathrm{~mL})$ and dry $\mathrm{Et}_{3} \mathrm{~N}$ $(3.4 \mathrm{~mL}, 24.4 \mathrm{mmol})$ was cooled at $0{ }^{\circ} \mathrm{C}$ and stirred with pivaloyl chloride $(1.8 \mathrm{~mL}, 14.5 \mathrm{mmol})$ and a catalytic amount of DMAP for $1 \mathrm{~h}$ at this temperature. The solution was transferred to a decantation funnel and the organic phase was consecutively washed with $1 \mathrm{~N} \mathrm{HCl}$, aqueous saturated $\mathrm{NaHCO}_{3}$ and aqueous saturated $\mathrm{NaCl}$, dried with $\mathrm{Na}_{2} \mathrm{SO}_{4}$, filtered, and concentrated to give a solid residue. Flash chromatography (eluent gradient: ethyl acetate/hexanes from $1: 9$ to $4: 6$ ) gave pure 6 (yield $1.83 \mathrm{~g}, 92 \%$ ). M.p. $38.3-38.8{ }^{\circ} \mathrm{C}$ (ethyl acetate/hexane). $[\alpha]_{\mathrm{D}}=56.2(c=0.26$, $\left.\mathrm{CHCl}_{3}\right) \cdot{ }^{1} \mathrm{H}$ NMR $\left(200 \mathrm{MHz}, \mathrm{CDCl}_{3}\right): \delta=7.24(\mathrm{~d}, J=9.0 \mathrm{~Hz}, 2$ $\mathrm{H}), 6.87(\mathrm{~d}, J=9.0 \mathrm{~Hz}, 2 \mathrm{H}), 5.12(\mathrm{~s}, 1 \mathrm{H}), 4.73(\mathrm{dd}, J=6$ and $4 \mathrm{~Hz}, 1 \mathrm{H}), 4.60$ (d, $J=11.0 \mathrm{~Hz}, 2 \mathrm{H}), 4.43(\mathrm{~d}, J=11.0 \mathrm{~Hz}, 2 \mathrm{H})$, $4.05(\mathrm{~m}, 2 \mathrm{H}), 3.83(\mathrm{dd}, J=5$ and $4 \mathrm{~Hz}, 1 \mathrm{H}), 3.8(\mathrm{~s}, 3 \mathrm{H}), 1.75$ $(\mathrm{m}, 4 \mathrm{H}), 1.46(\mathrm{~s}, 3 \mathrm{H}), 1.28(\mathrm{~s}, 3 \mathrm{H}), 1.20(\mathrm{~s}, 9 \mathrm{H}) .{ }^{13} \mathrm{C}$ NMR $\left(50 \mathrm{MHz}, \mathrm{CDCl}_{3}\right): \delta=178.3,159.2,129.6(2 \mathrm{C}), 129.1,113.5(2$ C), $112.4,104.5,85.2,82.0,80.1,69.4,66.5,63.9,55.0,38.5,29.1$, $26.8(3 \mathrm{C}), 25.7,24.6,24.2$. LRMS (EI): $m / z(\%)=452(0.16)\left[\mathrm{M}^{+}\right]$, 437 (0.3), $315(0.2), 299(0.3), 273(6.4), 258$ (1.4), 229 (3.8), 227 (1.8), 183 (4.5), 171 (3.6), 163 (1.8), 143 (1.6), 137 (2.1), 125 (10), 121 (100), 85 (7.2), 71 (10.3), 57 (32). $\mathrm{C}_{24} \mathrm{H}_{36} \mathrm{O}_{8}$ : calcd. C 63.70, H 8.02 ; found C 63.804 , H 8.022 .

6,7-Dideoxy-8-O-(2,2-dimethylpropanoyl)-2,3-O-(1-methylethylidene)- $\alpha$-D-manno-octofuranose (7): Alcohol $6(2 \mathrm{~g}, 4.4 \mathrm{mmol})$ in $\mathrm{CH}_{2} \mathrm{Cl}_{2}(90 \mathrm{~mL})$ and $\mathrm{H}_{2} \mathrm{O}(4.5 \mathrm{~mL})$ was vigorously stirred with $\operatorname{DDQ}(1.5 \mathrm{~g}, 6.6 \mathrm{mmol})$ at room temperature for $16 \mathrm{~h}$. Aqueous saturated $\mathrm{NaHCO}_{3}(1 \mathrm{~mL})$ was added and the resulting mixture stirred for $15 \mathrm{~min}$. Filtration through a pad of Celite and concentration gave a solid residue that was purified by flash chromatography (eluent gradient: ethyl acetate/hexanes from $2: 8$ to $6: 4$ ) to give pure hemiacetal 7 (yield $1.33 \mathrm{~g}, 91 \%$ ). M.p. $83.7-84.7{ }^{\circ} \mathrm{C}$ (ethyl acetate/hexane). $[\alpha]_{\mathrm{D}}=7.98\left(c=0.038, \mathrm{CHCl}_{3}\right) .{ }^{1} \mathrm{H}$ NMR $\left(200 \mathrm{MHz}, \mathrm{CDCl}_{3}\right): \delta=5.37(\mathrm{~s}, 1 \mathrm{H}), 4.69(\mathrm{dd}, J=6$ and $3 \mathrm{~Hz}, 1$ $\mathrm{H}), 4.57(\mathrm{~d}, J=6.0 \mathrm{~Hz}, 1 \mathrm{H}), 4.07$ (app. t, $2 \mathrm{H}, J=7$ and $6 \mathrm{~Hz})$, $3.97(\mathrm{~m}, 2 \mathrm{H}), 1.7(\mathrm{~m}, 4 \mathrm{H}), 1.41(\mathrm{~s}, 3 \mathrm{H}), 1.25(\mathrm{~s}, 3 \mathrm{H}), 1.17(\mathrm{~s}, 9$ $\mathrm{H}) .{ }^{13} \mathrm{C} \mathrm{NMR}\left(50 \mathrm{MHz}, \mathrm{CDCl}_{3}\right): \delta=178.6,112.3,100.5,85.8$, $82.9,79.1,69.7,64.0,38.6,28.6,26.7$ (3 C), 25.8, 24.6, 24.4. LRMS (EI): $m / z(\%)=317(55.5)\left[\mathrm{M}^{+}-15\right], 299(19.1), 255(5), 227(6.4)$, 215 (21.9), 212 (73.5), 202 (18.1), 197 (6.4), 189 (17.5), 183 (17.3), 173 (84.7), 171 (26), 159 (18), 155 (55.6), 143 (20.5), 137 (42.2), 131 (19.7), 129 (17.7), $126(46.7), 121$ (10.3), 114 (11.6), 113 (35.5), 109 (25.2). $\mathrm{C}_{16} \mathrm{H}_{28} \mathrm{O}_{7}$ : calcd. C 57.82, H 8.49; found C 57.994, H 8.58.

5,6-Dideoxy-7-O-(2,2-dimethylpropanoyl)-3-O-formyl-1,2-O-(1methylethylidene)- $\alpha$-D- $x y l o$-heptofuranose (8): Hemiacetal 7 (720 mg, $2.16 \mathrm{mmol})$ in dry $\mathrm{CH}_{2} \mathrm{Cl}_{2}(43 \mathrm{~mL})$ was heated at reflux with $\mathrm{I}_{2}(576 \mathrm{mg}, 2.3 \mathrm{mmol})$ and PhIO (freshly prepared) $(475 \mathrm{mg}$, $2.16 \mathrm{mmol})$ for $1 \mathrm{~h}$. More PhIO was added $(475 \mathrm{mg}, 2.16 \mathrm{mmol})$ and the heating continued for $1 \mathrm{~h}$ further. The same amount of $\mathrm{PhIO}$ was again added and the heterogeneous mixture was heated under reflux for further $3 \mathrm{~h}$. After cooling, the mixture was filtered through a pad of Celite, diluted with more dichloromethane, washed with aqueous $\mathrm{Na}_{2} \mathrm{~S}_{2} \mathrm{O}_{3}$ until complete decolouration was observed, dried with $\mathrm{Na}_{2} \mathrm{SO}_{4}$, and concentrated to give a gummy residue. Flash chromatography (eluent gradient: ethyl acetate/hexane from $1: 9$ to $4: 6$ ) gave pure 8 (yield $500 \mathrm{mg}, 70 \%$ ). $[\alpha]_{\mathrm{D}}=2.4$ $\left(c=0.287, \mathrm{CHCl}_{3}\right)$. IR $\left(\mathrm{CHCl}_{3}\right): \tilde{v}=1731,1724 \mathrm{~cm}^{-1} \cdot{ }^{1} \mathrm{H}$ NMR $\left(200 \mathrm{MHz}, \mathrm{CDCl}_{3}\right): \delta=8.07(\mathrm{~s}, 1 \mathrm{H}), 5.88(\mathrm{~d}, J=4.0 \mathrm{~Hz}, 1 \mathrm{H})$, $5.24(\mathrm{~d}, J=3.0 \mathrm{~Hz}, 1 \mathrm{H}), 4.50(\mathrm{~d}, J=4.0 \mathrm{~Hz}, 1 \mathrm{H}), 4.25(\mathrm{~m}, 1 \mathrm{H})$, 
4.04 (app. t, $J=6.0 \mathrm{~Hz}, 2 \mathrm{H}), 1.6(\mathrm{~m}, 4 \mathrm{H}), 1.49$ (s, $3 \mathrm{H}), 1.29$ (s, $3 \mathrm{H}), 1.16(\mathrm{~s}, 9 \mathrm{H}) .{ }^{13} \mathrm{C} \mathrm{NMR}\left(50 \mathrm{MHz}, \mathrm{CDCl}_{3}\right): \delta=178.3,159.5$, $111.8,104.2,83.3,78.3,75.4,63.7,38.6,27.1$ (3 C), 26.4, 26.0, 25.2, 24.5. LRMS (EI): $m / z(\%)=315(51.9)\left[\mathrm{M}^{+}-15\right], 227(30.3), 187$ (10.4), 125 (29.8), 121 (28.1), 107 (10.9), 97 (15), 85 (23), 71 (60.7), 57 (100). HRMS (EI): calcd. for $\mathrm{C}_{15} \mathrm{H}_{23} \mathrm{O}_{7}\left[\mathrm{M}^{+}-15\right] 315.144378$, found 315.144325 .

5,6-Dideoxy-7-O-(2,2-dimethylpropanoyl)-1,2-O-(1-methylethylidene)- $\alpha$-D- $x y l o$-heptofuranose (9): Formate 8 (800 mg, $2.4 \mathrm{mmol})$ in $\mathrm{Na}_{2} \mathrm{CO}_{3}$ saturated $\mathrm{MeOH}(25 \mathrm{~mL})$ was stirred for $10 \mathrm{~min}$. The solvent was evaporated and the residue dissolved in ethyl acetate, washed with aqueous saturated $\mathrm{NaCl}$, dried with $\mathrm{Na}_{2} \mathrm{SO}_{4}$, and concentrated to give the crude alcohol 9. Crystallization from ethyl acetate/hexane gave pure alcohol 9 (yield $812 \mathrm{mg}$, quant.). $[\alpha]_{\mathrm{D}}=14.6\left(c=0.268, \mathrm{CHCl}_{3}\right) . \operatorname{IR}\left(\mathrm{CHCl}_{3}\right): \tilde{v}=3500$, $1721 \mathrm{~cm}^{-1}$. ${ }^{1} \mathrm{H}$ NMR $\left(200 \mathrm{MHz}, \mathrm{CDCl}_{3}\right): \delta=5.90(\mathrm{~d}, J=4.0 \mathrm{~Hz}$, $1 \mathrm{H}), 4.51(\mathrm{~d}, J=4.0 \mathrm{~Hz}, 1 \mathrm{H}), 4.1(\mathrm{~m}, 3 \mathrm{H}), 1.8(\mathrm{~m}, 2 \mathrm{H}), 1.6(\mathrm{~m}$, $2 \mathrm{H}), 1.5(\mathrm{~s}, 3 \mathrm{H}), 1.31(\mathrm{~s}, 3 \mathrm{H}), 1.20(\mathrm{~s}, 9 \mathrm{H}) .{ }^{13} \mathrm{C} \mathrm{NMR}(50 \mathrm{MHz}$, $\left.\mathrm{CDCl}_{3}\right): \delta=178.8,111.3,104.2,85.3,79.9,75.1,64.2,29.7,27.1$ $(3 \mathrm{C}), 26.6,26.1,25.3,24.2$. LRMS (EI): $m / z(\%)=287(21)\left[\mathrm{M}^{+}\right.$ - 15], 227 (10.9), 173 (14), 159 (7.9), 143 (9.3), 125 (17.6), 100 (11.2), 85 (15.5), $71(100), 57(66.1), 55(10.5) \cdot \mathrm{C}_{15} \mathrm{H}_{26} \mathrm{O}_{6}$ : calcd. $\mathrm{C}$ $59.58, \mathrm{H} 8.67$; found C $59.385, \mathrm{H} 8.829$.

3-O-(tert-Butyldimethylsilyl)-5,6-dideoxy-7-O-(2,2-dimethylpropanoyl)-1,2-O-(1-methylethylidene)- $\alpha$-D-xylo-heptofuranose (10): Alcohol 9 (720 mg, $2.4 \mathrm{mmol})$ in dry DMF $(15 \mathrm{~mL})$ was stirred with imidazole $(326 \mathrm{mg}, 4.8 \mathrm{mmol})$ and TBDMSCl $(544 \mathrm{mg}$, $3.6 \mathrm{mmol}$ ) at $60^{\circ} \mathrm{C}$ for $6 \mathrm{~h}$. After cooling, diethyl ether was added and the mixture was transferred to a decantation funnel with the aid of more diethyl ether. The organic phase was washed with $\mathrm{H}_{2} \mathrm{O}$ $(3 \times)$, dried with $\mathrm{Na}_{2} \mathrm{SO}_{4}$, and concentrated to give an oily residue. Flash chromatography (eluent gradient: ethyl acetate/hexane from $5: 95$ to $2: 8$ ) gave pure 10 (yield $910 \mathrm{mg}, 91 \%)$. [ $\alpha]_{\mathrm{D}}=13.2(\mathrm{c}=$ $\left.0.205, \mathrm{CHCl}_{3}\right)$. IR $\left(\mathrm{CHCl}_{3}\right): \tilde{v}=1719 \mathrm{~cm}^{-1}$. ${ }^{1} \mathrm{H}$ NMR $(200 \mathrm{MHz}$, $\left.\mathrm{CDCl}_{3}\right): \delta=5.87(\mathrm{~d}, J=4.0 \mathrm{~Hz}, 1 \mathrm{H}), 4.36(\mathrm{~d}, J=4.0 \mathrm{~Hz}, 1 \mathrm{H})$, $4.07(\mathrm{~m}, 3 \mathrm{H}), 1.75(\mathrm{~m}, 2 \mathrm{H}), 1.49(\mathrm{~s}, 3 \mathrm{H}), 1.31(\mathrm{~s}, 3 \mathrm{H}), 1.19(\mathrm{~s}, 9$ $\mathrm{H}), .9(\mathrm{~s}, 9 \mathrm{H}), 0.12(\mathrm{~s}, 3 \mathrm{H}), 0.08(\mathrm{~s}, 3 \mathrm{H}) .{ }^{13} \mathrm{C} \mathrm{NMR}(50 \mathrm{MHz}$, $\left.\mathrm{CDCl}_{3}\right): \delta=178.5,111.3,104.6,85.6,80.6,76.4,64.3,38.7,27.2$ (3 C) $, 26.7,26.2,25.8(3 \mathrm{C}), 25.7,25.0,18.0,-4.7,-5.1$. LRMS (EI): $m / z(\%)=401(4.6)\left[\mathrm{M}^{+}-15\right], 359(3.9), 283(3.7), 215(6.9)$, 199 (29.2), 183 (9.3), 171 (5.6), 159 (100), 157 (17.4), 129 (74.1), 125 (9.3), 117 (5.4), 75 (22.3), 73 (28.5), 57 (51.9). $\mathrm{C}_{21} \mathrm{H}_{40} \mathrm{O}_{6} \mathrm{Si}$ : calcd. C 60.54, H 9.68; found C $60.22, \mathrm{H} 10.04$.

(1S)-1,4-Anhydro-2-O-(tert-butyldimethylsilyl)-5,6,7-trideoxy-1-[3(2,2-dimethylpropanoyloxy)propyl|-D-arabino-hept-6-enitol (11): The $1,2-O$-isopropylidene derivative $10(920 \mathrm{mg}, 2.2 \mathrm{mmol})$ in dry $\mathrm{CH}_{2} \mathrm{Cl}_{2}(20 \mathrm{~mL})$ was cooled to $0{ }^{\circ} \mathrm{C}$ and allyl trimethylsilane $(1.4 \mathrm{~mL}, 8.8 \mathrm{mmol})$ and boron trifluoride-diethyl ether (freshly distilled) $(0.6 \mathrm{~mL}, 4.7 \mathrm{mmol})$ were added. After $15 \mathrm{~min}$ at this temperature, the mixture was allowed to warm to room temperature and stirred for a further $6 \mathrm{~h}$. Aqueous saturated $\mathrm{NaHCO}_{3}$ was added and the organic phase decanted. The aqueous phase was extracted with more $\mathrm{CH}_{2} \mathrm{Cl}_{2}$, and the combined organic phases were washed with aqueous saturated $\mathrm{NaCl}$, dried with $\mathrm{Na}_{2} \mathrm{SO}_{4}$, and concentrated to give an oily residue. Flash chromatography (eluent gradient: ethyl acetate/hexane from $1: 9$ to $2: 8)$ gave pure alcohol 11 (yield $750 \mathrm{mg}, 85 \%) .[\alpha]_{\mathrm{D}}=13.8\left(c=0.42, \mathrm{CHCl}_{3}\right)$. IR $\left(\mathrm{CHCl}_{3}\right)$ : $\tilde{v}=3472,3023,1719, \mathrm{~cm}^{-1}$. The NMR data were determined for the acetate derivative. ${ }^{1} \mathrm{H}$ NMR $\left(500 \mathrm{MHz}, \mathrm{CDCl}_{3}\right): \delta=5.8(\mathrm{~m}, 1$ $\mathrm{H}) ; 5.08$ (ddd, $1 \mathrm{H}, J=17,2$ and $2 \mathrm{~Hz}$ ), $4.07(\mathrm{t}, J=6.0 \mathrm{~Hz}, 2 \mathrm{H}$ ), $3.93(\mathrm{dd}, J=3$ and $1 \mathrm{~Hz}, 1 \mathrm{H}), 3.84(\mathrm{ddt}, 1 \mathrm{H}, J=5,3$ and $2 \mathrm{~Hz}$ ), $3.81(\mathrm{td}, 1 \mathrm{H}, J=7$ and $2 \mathrm{~Hz}), 2.44(\mathrm{~m}, 1 \mathrm{H}), 2.07(\mathrm{~s}, 3 \mathrm{H})$, $1.77-1.57(\mathrm{~m}, 4 \mathrm{H}), 1.2(\mathrm{~s}, 9 \mathrm{H}), 0.9(\mathrm{~s}, 9 \mathrm{H}), 0.13(\mathrm{~s}, 3 \mathrm{H}), 0.06(\mathrm{~s}$, $3 \mathrm{H}) .{ }^{13} \mathrm{C} \mathrm{NMR}\left(125 \mathrm{MHz}, \mathrm{CDCl}_{3}\right): \delta=178.5,169.9,134.3,117.0$, 82.6 ( 2 C), 81.4, 76.8, 64.3, 38.6, 38.3, 27.1 (3 C), 25.8 (3 C), 25.7 (2 C) $, 20.9,18.0,-4.7,-5.5$. LRMS (EI): $m / z(\%)=343(2.4)\left[\mathrm{M}^{+}\right.$ $-\mathrm{C}_{4} \mathrm{H}_{9}$ ], $325(1), 299(0.6), 283(0.7), 257$ (1.6), 241 (3.2), 227 (2.6), 187 (7.7), 171 (12.4), 159 (100), 75 (15.2), 57 (53.1). $\mathrm{C}_{21} \mathrm{H}_{40} \mathrm{O}_{5} \mathrm{Si}$ : calcd. C $62.96, \mathrm{H} 10.06$; found C $63.36, \mathrm{H} 9.69$.

(1S)-1,4-Anhydro-2,3-bis- $O$-(tert-butyldimethylsilyl)-5,6,7-trideoxy1-[3-(2,2-dimethylpropanoyloxy)propyl]-D-arabino-hept-6-enitol (12): Alcohol $11(780 \mathrm{mg}, 1.95 \mathrm{mmol})$ in dry DMF was stirred with imidazole $(265 \mathrm{mg}, 3.9 \mathrm{mmol})$ and TBDMSCl $(4421 \mathrm{mg}, 2.9 \mathrm{mmol})$ at $60^{\circ} \mathrm{C}$ for $4 \mathrm{~h}$ and $30 \mathrm{~min}$. After cooling, diethyl ether was added and the mixture was transferred to a decantation funnel with the aid of more diethyl ether. The organic phase was washed with $\mathrm{H}_{2} \mathrm{O}$ $(3 \times)$, dried with $\mathrm{Na}_{2} \mathrm{SO}_{4}$, and concentrated to give an oily residue. Flash chromatography (eluent gradient: ethyl acetate/hexane from $5: 95$ to $6: 4)$ gave pure 12 (yield $970 \mathrm{mg}, 97 \%)$. [ $\alpha]_{\mathrm{D}}=12.3(\mathrm{c}=$ $\left.0.86, \mathrm{CHCl}_{3}\right)$. IR $\left(\mathrm{CHCl}_{3}\right): \tilde{v}=1719 \mathrm{~cm}^{-1} .{ }^{1} \mathrm{H} \mathrm{NMR}(500 \mathrm{MHz}$, $\left.\mathrm{CDCl}_{3}\right): \delta=5.8(\mathrm{~m}, 1 \mathrm{H}), 5.06(\mathrm{dd}, 1 \mathrm{H}, J=17$ and $2 \mathrm{~Hz}), 5.03$ $(\mathrm{d}, J=10.0 \mathrm{~Hz}, 1 \mathrm{H}), 4.06(\mathrm{t}, J=6.0 \mathrm{~Hz}, 2 \mathrm{H}), 3.92(\mathrm{~m}, 1 \mathrm{H})$, $3.83(\mathrm{~s}, 1 \mathrm{H}), 3.72(\mathrm{~s}, 1 \mathrm{H}), 3.71(\mathrm{t}, J=8.0 \mathrm{~Hz}, 1 \mathrm{H}), 2.39(\mathrm{~m}, 1$ H), $2.31(\mathrm{~m}, 1 \mathrm{H}), 1.8-1.54(\mathrm{~m}, 4 \mathrm{H}), 1.16(\mathrm{~s}, 9 \mathrm{H}), 0.89(\mathrm{~s}, 9 \mathrm{H})$, $0.85(\mathrm{~s}, 9 \mathrm{H}), 0.08(\mathrm{~s}, 3 \mathrm{H}), 0.06(\mathrm{~s}, 3 \mathrm{H}), 0.04(\mathrm{~s}, 3 \mathrm{H}), 0.02(\mathrm{~s}, 3$ $\mathrm{H}) .{ }^{13} \mathrm{C}$ NMR $\left(50 \mathrm{MHz}, \mathrm{CDCl}_{3}\right): \delta=178.5,135.2,116.9,86.2$, 81.1 (2 C), 79.5, 64.5, 38.7, 27.1 (3 C), 25.7 (2 C), 25.6 (3 C), -4.4, $-4.5(2 \mathrm{C}),-5.2$. LRMS (EI): $m / z(\%)=499(0.9)\left[\mathrm{M}^{+}-15\right], 473$ (2.4), 457 (24.2), 387 (4.6), 371 (3.8), 341 (14.5), 325 (10.8), 301 (8), 285 (16), 271 (3.9), $255(2.8), 239$ (11.1), 231 (4.6), 215 (15.4), 211 (18.9), 201 (18.9), $197(13.5), 189(1.7), 185(9.4), 171(3.7), 169$ $(2.1), 159$ (100), $157 / 6.9), 149$ (14.6), 147 (21.5), 141 (12.5), 133 (5.1), 129 (7.8), $115(8.7), 101$ (5.1), 89 (3.9), 75 (30.4), 73 (93.1), 57 (61.8). HRMS (EI): calcd. for $\mathrm{C}_{26} \mathrm{H}_{51} \mathrm{O}_{5} \mathrm{Si}_{2}\left[\mathrm{M}^{+}-15\right]$ 499.327507; found 499.328926 .

(7S)-7-Allyl-4,7-anhydro-5,6-bis- $O$-(tert-butyldimethylsilyl)-1,2,3trideoxy-D-arabino-hept-1-enitol (14): A solution of 12 (358 mg, $0.69 \mathrm{mmol}$ ) in dry $\mathrm{CH}_{2} \mathrm{Cl}_{2}$ was cooled to $-78^{\circ} \mathrm{C}$, and DIBAL $(0.9 \mathrm{~mL}, 0.9 \mathrm{mmol}, 1 \mathrm{M}$ in toluene) was added. The reaction mixture was stirred a $-78^{\circ} \mathrm{C}$ for $1 \mathrm{~h}$ and quenched with methanol (2-3 drops) and $\mathrm{H}_{2} \mathrm{O}$ and extracted with $\mathrm{CH}_{2} \mathrm{Cl}_{2}(15 \mathrm{~mL})$. The organic layer was dried with $\mathrm{Na}_{2} \mathrm{SO}_{4}$, and the solvent was removed under reduced pressure to give the alcohol 13 , which was used directly in the next synthetic step. - To a stirring THF solution of the alcohol $13(300 \mathrm{mg}, 0.7 \mathrm{mmol})$ and $o-\mathrm{O}_{2} \mathrm{NC}_{6} \mathrm{H}_{4} \mathrm{SeCN}(380 \mathrm{mg}$, $1.7 \mathrm{mmol})$ was added tributylphosphane $(0.4 \mathrm{~mL}, 1.67 \mathrm{mmol})$ dropwise, and the deep wine-red solution was stirred at room temperature for $16 \mathrm{~h}$, quenched with $\mathrm{H}_{2} \mathrm{O}$, and extracted with diethyl ether $(25 \mathrm{~mL})$. The organic layer was dried with $\mathrm{Na}_{2} \mathrm{SO}_{4}$, and the solvent was removed under reduced pressure. The residue was dissolved in THF $(9 \mathrm{~mL})$ at room temperature and then cooled to $0{ }^{\circ} \mathrm{C}$. To this solution was added $\mathrm{H}_{2} \mathrm{O}_{2}$, and the resulting mixture was stirred at $0^{\circ} \mathrm{C}$ for $3 \mathrm{~h}$, quenched with $\mathrm{H}_{2} \mathrm{O}(10 \mathrm{~mL})$, and extracted with diethyl ether $(20 \mathrm{~mL})$. The combined extracts were dried with $\mathrm{Na}_{2} \mathrm{SO}_{4}$, the solvent was removed under reduced pressure and the residue was purified by flash chromatography ( $n$-hexane/benzene: $7: 3$ ) to give 14 (yield $200 \mathrm{mg}, 75 \%$ for two steps) as a colourless oil. $[\alpha]_{\mathrm{D}}=+15.8\left(c=0.4, \mathrm{CHCl}_{3}\right)$. IR $\left(\mathrm{CHCl}_{3}\right): \tilde{v}=2954,2858,1643$, $1471,1257,1102 \mathrm{~cm}^{-1}$. ${ }^{1} \mathrm{H}$ NMR $\left(500 \mathrm{MHz}, \mathrm{CDCl}_{3}\right): \delta=5.58(\mathrm{~m}$, $4 \mathrm{H}$ ), (app. dq, $2 \mathrm{H}, J=17$ and $2 \mathrm{~Hz}$ ), 5.03 (dd, $2 \mathrm{H}, J=10$ and $1 \mathrm{~Hz}$ ), 3.97 (ddd, $1 \mathrm{H}, J=9,6$ and $3 \mathrm{~Hz}), 3.84(\mathrm{~s}, 1 \mathrm{H}), 3.75(\mathrm{~d}$, $J=4.0 \mathrm{~Hz}, 1 \mathrm{H}), 3.72$ (app. $\mathrm{t}, J=7.0 \mathrm{~Hz}, 1 \mathrm{H}), 2.44-2.3(\mathrm{~m}, 4$ $\mathrm{H}), 0.9(\mathrm{~s}, 9 \mathrm{H}), 0.84(\mathrm{~s}, 9 \mathrm{H}), 0.059(\mathrm{~s}, 3 \mathrm{H}),, 0.054(\mathrm{~s}, 3 \mathrm{H}), 0.049$ $(\mathrm{s}, 3 \mathrm{H}), 0.04(\mathrm{~s}, 3 \mathrm{H}) \cdot{ }^{13} \mathrm{C}$ NMR $\left(50 \mathrm{MHz}, \mathrm{CDCl}_{3}\right): \delta=135.4$, 
$135.2,116.8,86.2,81.0,80.9,79.1,76.9,38.6,33.5,25.6$ (3 C), 25.5 ( 3 C) $, 18.0,7.7,-4.3,-4.51,-4.55,-5.17$. HRMS (EI): calcd. for $\mathrm{C}_{22} \mathrm{H}_{44} \mathrm{O}_{3} \mathrm{Si}_{2} 412.3120$, found 412.3117 .

(7R)-4,7-Anhydro-7-(3-butenyl)-5,6-bis- $O$-(tert-butyldimethylsilyl)1,2,3-trideoxy-L-arabino-hept-1-enitol (15): DMSO $(0.07 \mathrm{~mL}$, $0.96 \mathrm{mmol}$ ) was added dropwise to a solution of oxalyl chloride $(0.04 \mathrm{~mL}, 0.48 \mathrm{mmol})$ in $\mathrm{CH}_{2} \mathrm{Cl}_{2}(2 \mathrm{~mL})$ at $-78{ }^{\circ} \mathrm{C}$, and the resulting mixture was stirred for $20 \mathrm{~min}$. A solution of alcohol $\mathbf{1 3}$ (see above) (104 mg, $0.24 \mathrm{mmol})$ in $\mathrm{CH}_{2} \mathrm{Cl}_{2}(2 \mathrm{~mL})$ was added via cannula to the reaction mixture and the resulting mixture was stirred for $20 \mathrm{~min}$ at this temperature. Then, $\mathrm{Et}_{3} \mathrm{~N}(0.2 \mathrm{~mL}$, $1.44 \mathrm{mmol}$ ) was added and the resulting mixture stirred for $10 \mathrm{~min}$ before being warmed to room temperature. The mixture was poured into $\mathrm{H}_{2} \mathrm{O}$ and the two layers were separated. The aqueous layer was extracted with diethyl ether $(2 \times 2 \mathrm{~mL})$ and the combined organic layers were dried with $\mathrm{Na}_{2} \mathrm{SO}_{4}$. The solvent was removed under reduced pressure, and the crude aldehyde was used in the next reaction without further purification. - A suspension of methyltriphenylphosphonium iodide $(126 \mathrm{mg}, 0.3 \mathrm{mmol})$ in THF $(1.5 \mathrm{~mL})$ was treated at $0{ }^{\circ} \mathrm{C}$ with $n \mathrm{BuLi}(0.2 \mathrm{~mL}, 0.3 \mathrm{mmol}, 1.6 \mathrm{~N}$ in hexane). After stirring for $1 \mathrm{~h}$ at this temperature, the aldehyde (the residue from the previous step) in dry THF $(1.5 \mathrm{~mL})$ was added via cannula. After $1 \mathrm{~h}$, the reaction was quenched with aqueous saturated $\mathrm{NH}_{4} \mathrm{Cl}$ solution ( $1 \mathrm{~mL}$ ), and extracted with diethyl ether $(2 \times 3 \mathrm{~mL})$. The organic layer was dried with $\mathrm{Na}_{2} \mathrm{So}_{4}$, the solvent was removed under reduced pressure and the residue purified by flash chromatography ( $n$-hexane/benzene: 7:3) to give $\mathbf{1 5}$ (yield $72 \mathrm{mg}, 70 \%$ for the two steps) as a colourless oil. $[\alpha]_{\mathrm{D}}=+22.5$ $\left(c=0.14, \mathrm{CHCl}_{3}\right)$. IR $\left(\mathrm{CHCl}_{3}\right): \tilde{v}=3018,2956,2930,2858,1640$, $1471,1463,1258 \mathrm{~cm}^{-1}$. ${ }^{1} \mathrm{H}$ NMR $\left(500 \mathrm{MHz}, \mathrm{CDCl}_{3}\right): \delta=5.8(\mathrm{~m}$, $4 \mathrm{H}), 5.0(\mathrm{~m}, 2 \mathrm{H}), 3.91$ (ddd, $1 \mathrm{H}, J=9,8$ and $3 \mathrm{~Hz}$ ), $3.83(\mathrm{~s}, 1$ $\mathrm{H}), 3.75(\mathrm{~d}, J=3.0 \mathrm{~Hz}), 3.7(\mathrm{~d}, J=7.0 \mathrm{~Hz}, 1 \mathrm{H}), 2.4(\mathrm{~m}, 1 \mathrm{H})$, $2.3(\mathrm{~m}, 1 \mathrm{H}), 2.16(\mathrm{~m}, 1 \mathrm{H}), 2.07(\mathrm{~m}, 1 \mathrm{H}), 1.73(\mathrm{~m}, 1 \mathrm{H}), 1.62(\mathrm{~m}$, $1 \mathrm{H}), 0.89$ (s, $9 \mathrm{H}), 0.08(\mathrm{~s}, 3 \mathrm{H}), 0.06(\mathrm{~s}, 3 \mathrm{H}), 0.005(\mathrm{~s}, 3 \mathrm{H}), 0.004$ $(\mathrm{s}, 3 \mathrm{H}), 0.002(\mathrm{~s}, 3 \mathrm{H}) .{ }^{13} \mathrm{C}$ NMR $\left(50 \mathrm{MHz}, \mathrm{CDCl}_{3}\right): \delta=138.4$, $135.2,116.7,114.2,86.0,89.0$ (2 C), 79.2, 38.6, 30.5, 28.1, 25.7 (3 C), 25.5 (3 C), 17.9, 17.7, -4.3, -4.5, $-4.54,-5.1$. HRMS (EI): calcd. for $\mathrm{C}_{23} \mathrm{H}_{46} \mathrm{O}_{3} \mathrm{Si}_{2} 426.298553$; found 426.297256 .

(7R)-4,7-Anhydro-5,6-bis- $O$-(tert-butyldimethylsilyl)-7-(3-cyanopropyl)-1,2,3-trideoxy-L-arabino-hept-1-enitol (17): To a solution of alcohol $13(207 \mathrm{mg}, 0.48 \mathrm{mmol})$ in dry $\mathrm{CH}_{2} \mathrm{Cl}_{2}(5 \mathrm{~mL})$ was added dry $\mathrm{Et}_{3} \mathrm{~N}(0.33 \mathrm{~mL}, 2.4 \mathrm{mmol})$ and $\mathrm{MsCl}(0.07 \mathrm{~mL}, 0.96 \mathrm{mmol})$ and the resulting mixture was stirred at $0{ }^{\circ} \mathrm{C}$ for $30 \mathrm{~min}$. More $\mathrm{CH}_{2} \mathrm{Cl}_{2}$ was added and the resulting solution was washed with $1 \mathrm{~N} \mathrm{HCl}$. The organic layer was dried with $\mathrm{Na}_{2} \mathrm{So}_{4}$ and the solvent removed under reduced pressure. The crude mesylate $\mathbf{1 6}$ was dissolved in dry DMF $(3 \mathrm{~mL})$ and stirred after addition of $\mathrm{KCN}(274 \mathrm{mg}$, $4.21 \mathrm{mmol}$ ) at $50{ }^{\circ} \mathrm{C}$ for $10 \mathrm{~h}$. Then, the reaction mixture was allowed to cool, quenched with $\mathrm{H}_{2} \mathrm{O}(10 \mathrm{~mL})$, and extracted with diethyl ether $(2 \times 10 \mathrm{~mL})$. The organic layer was dried with $\mathrm{Na}_{2} \mathrm{SO}_{4}$, the solvent was removed under reduce pressure and the residue was purified by flash chromatography (ethyl acetate/hexane: $10: 90)$ to give the cyano derivative $17(126 \mathrm{mg}, 68 \%)$ as a colourless oil. $[\alpha]_{\mathrm{D}}=+18.1\left(c=0.22, \mathrm{CHCl}_{3}\right)$. IR $\left(\mathrm{CHCl}_{3}\right): \tilde{v}=3079,2955$, $2930,2858,2249,1641,1471,1361,1257,1111,1078 \mathrm{~cm}^{-1} .{ }^{1} \mathrm{H}$ NMR $\left(200 \mathrm{MHz}, \mathrm{CDCl}_{3}\right): \delta=5.01(\mathrm{~m}, 1 \mathrm{H}), 5.12(\mathrm{~m}, 2 \mathrm{H}), 3.93$ $(\mathrm{m}, 1 \mathrm{H}), 3.84(\mathrm{~s}, 1 \mathrm{H}), 3.74(\mathrm{~m}, 2 \mathrm{H}), 2.38(\mathrm{~m}, 4 \mathrm{H}), 1.79(\mathrm{~m}, 4$ $\mathrm{H}), 0.91(\mathrm{~s}, 9 \mathrm{H}), 0.88(\mathrm{~s}, 9 \mathrm{H}), 0.09(\mathrm{~s}, 3 \mathrm{H}), 0.07(\mathrm{~s}, 3 \mathrm{H}), 0.06(\mathrm{~s}$, $3 \mathrm{H}), 0.02(\mathrm{~s}, 3 \mathrm{H}) .{ }^{13} \mathrm{C} \mathrm{NMR}\left(50 \mathrm{MHz}, \mathrm{CDCl}_{3}\right): \delta=135.1,116.9$, $86.3,80.9,80.5,79.7,38.6,28.4$ (2 C), 25.7 (3 C), 25.7 (3 C), 22.8, $17.9,17.7,17.2,-4.3,-4.4,-4.5,-5.0$. HRMS (EI): calcd. for $\mathrm{C}_{22} \mathrm{H}_{42} \mathrm{NO}_{3} \mathrm{Si}_{2}\left[\mathrm{M}^{+}-15\right] 424.270326$; found 424.266037 .
(7R)-4,7-Anhydro-5,6-bis- $O$-(tert-butyldimethylsilyl)-1,2,3-trideoxy7-(4-pentenyl)-L-arabino-hept-1-enitol (18): A solution of 17 (80 mg, $0.18 \mathrm{mmol})$ in dry $\mathrm{CH}_{2} \mathrm{Cl}_{2}(3 \mathrm{~mL})$ was cooled to $-78^{\circ} \mathrm{C}$ and DIBAL $(0.7 \mathrm{~mL}, 0.7 \mathrm{mmol}, 1 \mathrm{M}$ in toluene $)$ was added. The reaction mixture was stirred at $-78^{\circ} \mathrm{C}$ for $1 \mathrm{~h}$ and quenched with $1 \mathrm{~N} \mathrm{HCl}$ $(2 \mathrm{~mL})$. The cooling bath was removed and the mixture was stirred for $1 \mathrm{~h}$ and extracted with $\mathrm{CH}_{2} \mathrm{Cl}_{2}(2 \times 10 \mathrm{~mL})$. The organic layer was dried with $\mathrm{Na}_{2} \mathrm{SO}_{4}$, the solvent was removed under reduced pressure and the crude aldehyde residue was directly used in the Wittig reaction. - A suspension of methyltriphenylphosphonium iodide $(124 \mathrm{mg}, 0.31 \mathrm{mmol})$ in THF $(1.5 \mathrm{~mL})$ was treated at $0{ }^{\circ} \mathrm{C}$ with $n \mathrm{BuLi}(0.3 \mathrm{~mL}, 0.3 \mathrm{mmol}, 1.0 \mathrm{~N}$ in hexanes). After stirring for $1 \mathrm{~h}$ at this temperature, the aldehyde (the crude residue from the previous step), dissolved in dry THF $(1.5 \mathrm{~mL})$, was added via cannula. After $1 \mathrm{~h}$, the reaction was quenched with aqueous saturated $\mathrm{NH}_{4} \mathrm{Cl}$ solution $(2 \mathrm{~mL})$ and extracted with diethyl ether $(2 \times$ $4 \mathrm{~mL}$ ). The organic layer was dried with $\mathrm{Na}_{2} \mathrm{SO}_{4}$, the solvent was removed under reduced pressure and the residue was purified by flash chromatography ( $n$-hexane/benzene: 6:4) to give $\mathbf{1 8}$ (yield $45 \mathrm{mg}, 56 \%$ for the two steps $)$ as a colourless oil. $[\alpha]_{\mathrm{D}}=+10(c=$ $\left.0.1, \mathrm{CHCl}_{3}\right)$. IR $\left(\mathrm{CHCl}_{3}\right): \tilde{v}=3077,3018,2955,2930,2858,1642$, $1474,1361,1257,1220,1101,1005 \mathrm{~cm}^{-1}$. ${ }^{1} \mathrm{H}$ NMR $(500 \mathrm{MHz}$, $\left.\mathrm{CDCl}_{3}\right): \delta=5.8(\mathrm{~m}, 2 \mathrm{H}), 5.0(\mathrm{~m}, 4 \mathrm{H}), 3.99(\mathrm{ddd}, 1 \mathrm{H}, J=8,5$ and $3 \mathrm{~Hz}), 3.83(\mathrm{~s}, 1 \mathrm{H}), 3.71-3.69(\mathrm{~m}, 2 \mathrm{H}), 2.4(\mathrm{~m}, 1 \mathrm{H}), 2.3(\mathrm{~m}$, $1 \mathrm{H}), 2.1(\mathrm{~m}, 2 \mathrm{H}), 1.63(\mathrm{~m}, 1 \mathrm{H}), 1.54(\mathrm{~m}, 1 \mathrm{H}), 1.4-1.23(\mathrm{~m}, 2$ H), 0.89 (s, $9 \mathrm{H}), 0.86$ (s, $9 \mathrm{H}), 0.07$ (s, $3 \mathrm{H}), 0.04$ (s, $3 \mathrm{H}), 0.03$ (s, $3 \mathrm{H}), 0.02(\mathrm{~s}, 3 \mathrm{H}) \cdot{ }^{13} \mathrm{C} \mathrm{NMR}\left(50 \mathrm{MHz}, \mathrm{CDCl}_{3}\right): \delta=138.6,135.2$, $116.2,114.4,86.0,81.5,81.0,79.3,38.6,34.0,28.6(2 \mathrm{C}), 25.7$ (3 C), 25.6 (3 C), 16.0, 17.7, $-4.3,-4.46,-4.5,-5.1$. HRMS (EI): calcd. for $\mathrm{C}_{24} \mathrm{H}_{48} \mathrm{O}_{3} \mathrm{Si}_{2} 440.314203$; found 440.318733 .

$\{\mid(3 \mathrm{a} R, 5 R, 6 S, 6 \mathrm{a} R)-5$-(3-Butenyl)-2,2-dimethyltetrahydrofuro|2,3- $d]$ [1,3]dioxol-6-yl]oxy\}(tert-butyl)dimethylsilane (22): To a slurry of 3$O$-(tert-butyldimethylsilyl)-1,2-O-isopropylidene- $\alpha$-D- $x y$ lo-furanose (20) $(500 \mathrm{mg}, 1.6 \mathrm{mmol})$ in dry benzene $(15 \mathrm{~mL})$ was added imidazole (148 mg, $2.13 \mathrm{mmol})$ and $\mathrm{Ph}_{3} \mathrm{P}(558 \mathrm{mg}, 2.13 \mathrm{mmol})$. The mixture was then cooled to $0{ }^{\circ} \mathrm{C}$ and iodine $(541 \mathrm{mg}, 2.13 \mathrm{mmol})$ was added. After stirring for $2 \mathrm{~h}$, the mixture was filtered through a pad of Celite and concentrated to give a gummy residue. Flash chromatography (ethyl acetate/hexane: 1:9) gave pure iodine derivative 21 (yield $638 \mathrm{mg}, 90 \%$ ), which was taken in dry toluene $(15 \mathrm{~mL})$ and heated at reflux with allyltributyltin $(1.9 \mathrm{~mL}$, $6.16 \mathrm{mmol}$ ) and a catalytic amount of AIBN overnight. The solvent was distilled off and the gummy residue purified by flash chromatography (ethyl acetate/hexane: 1:9) to give pure 22 (yield $389 \mathrm{mg}$, $86 \%) .[\alpha]_{\mathrm{D}}=-13.8\left(c=0.36, \mathrm{CHCl}_{3}\right)$. IR $\left(\mathrm{CHCl}_{3}\right): \tilde{v}=3067$, $1639 \mathrm{~cm}^{-1}$. ${ }^{1} \mathrm{H}$ NMR $\left(200 \mathrm{MHz}, \mathrm{CDCl}_{3}\right): \delta=5.84(\mathrm{~d}, J=4.0 \mathrm{~Hz}$, $1 \mathrm{H}), 5.85-5.75(\mathrm{~m}, 1 \mathrm{H}), 5.01$ (app. dq, $1 \mathrm{H}, J=17$ and $2 \mathrm{~Hz}$ ), 4.94 (app. dd, $J=10$ and $1 \mathrm{~Hz}, 1 \mathrm{H}), 4.32(\mathrm{~d}, J=4.0 \mathrm{~Hz}, 1 \mathrm{H}$ ), 4.08 (ddd, $1 \mathrm{H}, J=8,6$ and $3 \mathrm{~Hz}), 4.0(\mathrm{~d}, J=3.0 \mathrm{~Hz}, 1 \mathrm{H}), 2.16$ $(\mathrm{m}, 1 \mathrm{H}), 2.08(\mathrm{~m}, 1 \mathrm{H}), 1.7(\mathrm{~m}, 1 \mathrm{H}), 1.6(\mathrm{~m}, 1 \mathrm{H}), 1.45(\mathrm{~s}, 3 \mathrm{H})$, $1.28(\mathrm{~s}, 3 \mathrm{H}), 0.86(\mathrm{~s}, 9 \mathrm{H}), 0.1(\mathrm{~s}, 3 \mathrm{H}), 0.06(\mathrm{~s}, 3 \mathrm{H}) .{ }^{13} \mathrm{C}$ NMR $\left(50 \mathrm{MHz}, \mathrm{CDCl}_{3}\right): \delta=138.0,114.7,111.2,104.4,88.6,86.3,71.1$, $30.2,27.3,26.7,27.3$ (3 C), 18.0, -4.6, -8.1. HRMS (EI): calcd. for $\mathrm{C}_{15} \mathrm{H}_{26} \mathrm{O}_{4} \mathrm{Si}_{2}\left[\mathrm{M}^{+}-15\right]$ 313.183513; found 313.185493 .

(1R)-1,4-Anhydro-1-(3-butenyl)-2,3-bis- $O$-(tert-butyldimethylsilyl)5,6,7-trideoxy-L-arabino-hept-6-enitol (ent-15): The 1,2-O-isopropylidene derivative $22(977 \mathrm{mg}, 2.97 \mathrm{mmol})$ in dry $\mathrm{CH}_{2} \mathrm{Cl}_{2}(36 \mathrm{~mL})$ was cooled to $0{ }^{\circ} \mathrm{C}$, and allyltrimethylsilane $(1.87 \mathrm{~mL}, 11.8 \mathrm{mmol})$ and boron trifluoride-diethyl ether (freshly distilled) $(0.75 \mathrm{~mL}$, $5.95 \mathrm{mmol}$ ) were added. After $15 \mathrm{~min}$ at this temperature, the mixture was allowed to warm to room temperature and stirred overnight. Aqueous saturated $\mathrm{NaHCO}_{3}$ solution was added and the 
organic phase decanted. The aqueous phase was extracted with more $\mathrm{CH}_{2} \mathrm{Cl}_{2}$, and the combined organic phases were washed with aqueous saturated $\mathrm{NaCl}$ solution, dried with $\mathrm{Na}_{2} \mathrm{SO}_{4}$ and concentrated to give an oily residue. Flash chromatography (eluent gradient: ethyl acetate/benzene from 5:95 to 1:9) gave the pure alcohol (yield $515 \mathrm{mg}, 55 \%$ ) which was transformed into the diene ent-15 $\left\{[\alpha]_{\mathrm{D}}=-19\left(c=0.2, \mathrm{CHCl}_{3}\right)\right\}$ using the same procedure as in the case of 12 (see above).

Synthesis of the Oxabicycles 23, 24, 25, and ent-24. - Method A: To a solution of the corresponding dienes 14, 15, 18, and ent-15 $(0.23 \mathrm{mmol}, 0.02 \mathrm{M})$ in dry degassed benzene was added the Grubbs' catalyst $(20 \mathrm{mmol} \%)$. After stirring the reaction mixture for $16 \mathrm{~h}$ at reflux, the solvent was removed in vacuo and the residue was purified by flash chromatography ( $n$-hexane/benzene: $7: 3$ ) to give the corresponding oxabicycles $23,24,25$, and $\boldsymbol{e n t}$-24, respectively. - Method B: The same as method A, but using more dilute conditions $(0.003 \mathrm{M}$ in diene). - Method C: To a solution of the corresponding dienes $(0.23 \mathrm{mmol}, 0.02 \mathrm{M})$ in dry degassed benzene was added the Grubbs' catalyst $(10 \mathrm{mmol} \%)$. After stirring the reaction mixture for $8 \mathrm{~h}$ at reflux, a second charge of catalyst was added $(10 \mathrm{mmol} \%)$ and the mixture further heated under reflux for $8 \mathrm{~h}$. The solvent was removed in vacuo and the residue was purified by flash chromatography ( $n$-hexane/benzene: $7: 3$ ) as in method A.

tert-Butyl( $\{(1 S, 6 R, 7 S, 8 R)-8$-I(tert-butyldimethylsilyl)oxy\}-9-oxabicyclo[4.2.1]non-3-en-7-yl\}oxy)dimethylsilane $(23):[\alpha]_{\mathrm{D}}=+13.3(c=$ $\left.0.38, \mathrm{CHCl}_{3}\right)$. IR $\left(\mathrm{CHCl}_{3}\right): \tilde{v}=3013,2956,2930,2857,2358,1711$, $1471,1252,1103 \mathrm{~cm}^{-1} .{ }^{1} \mathrm{H}$ NMR $\left(500 \mathrm{MHz}, \mathrm{CDCl}_{3}\right): \delta=5.6-5.5$ $(\mathrm{m}, 2 \mathrm{H}), 4.26-4.23(\mathrm{~m}, 2 \mathrm{H}), 4.0-3.98(\mathrm{~m}, 2 \mathrm{H}), 2.48(\mathrm{~m}, 1 \mathrm{H})$, $2.35-2.27(\mathrm{~m}, 3 \mathrm{H}), 0.86(\mathrm{~s}, 18 \mathrm{H}), 0.06(\mathrm{~s}, 3 \mathrm{H}), 0.05(\mathrm{~s}, 3 \mathrm{H}), 0.03$ (s, $3 \mathrm{H}) .{ }^{13} \mathrm{C} \mathrm{NMR}\left(125 \mathrm{MHz}, \mathrm{CDCl}_{3}\right): \delta=129.2,125.2,83.7,83.4$, $82.0,78.9,36.2,32.2,25.7,17.2$ (2 C), $-4.8,-4.6,-4.7,-4.1$. $\mathrm{C}_{20} \mathrm{H}_{40} \mathrm{O}_{3} \mathrm{Si}_{2}$ : calcd. C 62.44, $\mathrm{H} \mathrm{10.48;} \mathrm{found} \mathrm{C} 62.14, \mathrm{H} 10.64$

tert-Butyl( $\{(1 S, 7 R, 8 S, 9 R)-9$-[(tert-butyldimethylsilyl)oxy]-10-oxabicyclo[5.2.1]dec-4-en-8-yl\}oxy)dimethylsilane (24): $[\alpha]_{\mathrm{D}}=+4.3(\mathrm{c}=$ $\left.0.42, \mathrm{CHCl}_{3}\right)$. IR $\left(\mathrm{CHCl}_{3}\right): \tilde{v}=3012,2959,2934,2928,2859,2854$, $1471,1463,1361,1257,1210,1101,1083 \mathrm{~cm}^{-1}$. ${ }^{1} \mathrm{H}$ NMR $\left(500 \mathrm{MHz}, \mathrm{CDCl}_{3}\right): \delta=5.82-5.53(\mathrm{~m}, 2 \mathrm{H}), 4.05$ (app. d, $J=$ $8.0 \mathrm{~Hz}, 1 \mathrm{H}), 3.96-3.94(\mathrm{~m}, 1 \mathrm{H}), 3.81-3.76(\mathrm{~m}, 2 \mathrm{H}), 2.52-2.46$ $(\mathrm{m}, 1 \mathrm{H}), 2.45-2.22(\mathrm{~m}, 3 \mathrm{H}), 1.7-1.62(\mathrm{~m}, 2 \mathrm{H}), 0.9(\mathrm{~s}, 3 \mathrm{H}), 0.87$ $(\mathrm{s}, 9 \mathrm{H}), 0.08(\mathrm{~s}, 3 \mathrm{H}), 0.062(\mathrm{~s}, 3 \mathrm{H}), 0.05(\mathrm{~s}, 3 \mathrm{H}), 0.04(\mathrm{~s}, 3 \mathrm{H})$. ${ }^{13} \mathrm{C}$ NMR $\left(125 \mathrm{MHz}, \mathrm{CDCl}_{3}\right): \delta=131.3,127.6,88.0,83.6,82.2$, $79.5,32.5$ (2 C), 27.0, 26.0, 25.8, 18.1, 18.0, -4.3, -4.6, -4.95, -4.99 . $\mathrm{C}_{21} \mathrm{H}_{42} \mathrm{O}_{3} \mathrm{Si}_{2}$ : calcd. C $63.26, \mathrm{H} 10.61$; found $\mathrm{C} 62.9, \mathrm{H}$ 10.21 .

ent-24: $[\alpha]_{D}=-5.5\left(c=0.18, \mathrm{CHCl}_{3}\right)$.

tert-Butyl $(\{(1 S, 7 R, 8 S, 9 R)-9-[($ tert-butyldimethylsilyl)oxyl-10-oxabicyclo[5.2.1]dec-4-en-8-yl\}oxy)dimethylsilane (25): $[\alpha]_{\mathrm{D}}=+10.9(c=$ $\left.0.22, \mathrm{CHCl}_{3}\right)$. IR $\left(\mathrm{CHCl}_{3}\right): \tilde{v}=3014,2960,2929,2850,2350,1420$, $1237 \mathrm{~cm}^{-1} .{ }^{1} \mathrm{H}$ NMR $\left(500 \mathrm{MHz}, \mathrm{CDCl}_{3}\right): \delta=5.64-5.52(\mathrm{~m}, 2 \mathrm{H})$, 4.03 (app. d, $J=9.0 \mathrm{~Hz}, 1 \mathrm{H}), 3.94-3.89(\mathrm{~m}, 1 \mathrm{H}), 3.77-3.75(\mathrm{~m}$, $2 \mathrm{H}), 2.4-2.0(\mathrm{~m}, 6 \mathrm{H}), 1.7-1.5(\mathrm{~m}, 2 \mathrm{H}), 0.89(\mathrm{~s}, 9 \mathrm{H}), 0.87(\mathrm{~s}, 9$ $\mathrm{H}), 0.09(\mathrm{~s}, 3 \mathrm{H}), 0.088(\mathrm{~s}, 3 \mathrm{H}), 0.08(\mathrm{~s}, 3 \mathrm{H}), 0.06(\mathrm{~s}, 3 \mathrm{H}) .{ }^{13} \mathrm{C}$ NMR $\left(125 \mathrm{MHz}, \mathrm{CDCl}_{3}\right): \delta=131.1,127.9,88.0,83.4,81.1,79.5$, $29.4,28.8$ (2 C), 25.9, 25.7, 23.6, 18.0, 17.8, -4.4, -4.5, -4.7, -5.0. $\mathrm{C}_{22} \mathrm{H}_{44} \mathrm{O}_{3} \mathrm{Si}_{2}$ : calcd. C $64.02, \mathrm{H} 10.74$; found $\mathrm{C} 63.64, \mathrm{H}$ 10.75 .

\section{Acknowledgments}

We gratefully acknowledge financial support by the Spanish Ministerio de Ciencia y Tecnología (PB98-0443-C02-02), the Canary Islands Consejería de Educación y Ciencia (COFI2000/03), and the FEDER (1SD97-0747-C4-01).

[1] [1a] F. Fürstner, Angew, Chem. Int. Ed. 2000, 39, 3012. ${ }^{[\mathrm{lb}]} \mathrm{A}$ Fürstner, Top. Catal. 1997, 4, 285. ${ }^{[1 \mathrm{c}]}$ M. Schuster; S. Blechert, Angew. Chem. Int. Ed. Engl. 1997, 36, 2036. ${ }^{[1 d]} \mathrm{R}$. H Grubbs, S. Chang, Tetrahedron 1998, 54, 4413. [le] S. K Armstrong, $J$. Chem. Soc., Perkin Trans. 1 1998, 371.

[2] For recent studies of asymmetric ring-closing metathesis, see: [2a] J. B. Alexander, D. S. La, D. R. Cefalo, A. H. Hoveyda, R. R. Schrock, J. Am. Chem. Soc. 1998, 120, 4041 and references cited therein. ${ }^{[2 b]}$ D. S. La, J. B. Alexander, D. R. Cefalo, D. D. Craf, A. H. Hoveyda, R. R. Schrock, J. Am. Chem. Soc. 1998, 120,9720 .

[3] [3a] P. Schwab, M. B. France, J. W. Ziller, R. H. Grubbs, Angew. Chem. Int. Ed. Engl. 1995, 34, 2039. [3b] P. Schwab, R. H Grubbs, J. W.Ziller, J. Am. Chem. Soc. 1996, 118, 100.

[4] For synthesis of small bridged bicyclo-alkanes, see: ${ }^{44 a]} \mathrm{A}$. Morehead, R. H. Grubbs, Chem. Commun. 1998, 275. ${ }^{[4 b]}$ J. L. Wood, A. H. Alexandra, B. M. Stoltz, M. M. Weiss, J. A. Dixon, B. D. Doan, M. F. Shamji, J. M. Chen, T. P. Heffron, J. Am. Chem. Soc. 1999, 121, 6326. ${ }^{[4 \mathrm{c}]}$ M. Wenz, D. Großbach, M. Beitzel, S. Blechert, Synthesis 1999, 607. For synthesis of medium bridged-oxabicycloalkanes, see: [4d] I. Hanna, V. Michaut, Org. Lett. 2000, 2, 1141.

[5] For a recent review, see: P. Chiu, M. Lautens, Top. Curr: Chem. 1997, 190, 1.

[6] For a review of these compounds with a survey of their biological activities, see: P. Bernardelli, L. A. Paquette, Heterocycles 1998, 49, 531.

[7] We have recently completed an in-depth study of the template effect of this bicyclic acetal on the stereoselective synthesis of C-furanosides: F. García-Tellado, P. Armas, J. J. Marrero-Tellado, Angew. Chem, Int. Ed. Engl. 2000, 30, 2727. For stereoselective allylations of five-membered ring oxocarbenium ions, see: ${ }^{[7 a]}$ C. H. Larsen, B. H. Ridway, J. T. Shaw, K. A. Woerpel, J. Am. Chem. Soc. 1999, 121, 12208. ${ }^{[7 \mathrm{~b}]}$ J. T. Shaw, K. A. Woerpel, Tetrahedron 1999, 55, 8747. ${ }^{[7 \mathrm{c}]}$ A. Schmitt, H.-U. Reissig, Eur. J. Org. Chem. 2001, 1169. ${ }^{[7 \mathrm{~d}]}$ A. Schmitt, H.-U. Reissig, Eur. J. Org. Chem. 2000, 3893. ${ }^{[7 \mathrm{e}]}$ A. Schmitt, H.-U. Reissig, Chem Ber 1995, 128, 871. ${ }^{[7]}$ A. Schmitt, H.-U. Reissig, Synlett 1990, 40. [7g] J. Ishihara, J. Miyakawa, T. Tsujimoto, A. Murai, Synlett 1997, 1417.

[8] P. Armas, C. G. Francisco, E. Súarez, J. Am. Chem. Soc. 1993, 113,8865

[9] Although the reaction was initially described as a radical process, we have shown that the reaction is ionic in nature: P. Armas, F. García-Tellado, J. J. Marrero-Tellado, J. Robles, Tetrahedron Lett. 1997, 38, 8081.

[10] M. E. Evans, F. W. Parrish, Methods Carbohydr. Chem. 1980, 8, 173.

[11] B. Lipshutz, in Organometallics in Synthesis (Ed.: M. Schlosser), John Wiley, New York, 1994, chapter 4.

[12] P. B. Alper, M. Hendrix, P. Sears, C.-H. Wong, J. Am. Chem. Soc. 1998, 120, 1965.

[13] M. Ulman, R. H. Grubbs, J. Org. Chem. 1999, 64, 7202.

[14] [14a] M. Scholl, T. M. Trnka, J. P. Morgan, R. H. Grubbs, Tetrahedron Lett. 1999, 40, 2247. ${ }^{[14 \mathrm{~b}]}$ See ref. ${ }^{[1 \mathrm{a}]}$

Received June 8, 2001

[O01276] 